University of Nebraska - Lincoln

DigitalCommons@University of Nebraska - Lincoln

Robert G. Fuller Publications and Presentations Research Papers in Physics and Astronomy

January 1969

\title{
Equilibrium concentration of impurity-vacancy complexes
}

Robert Fuller

rfuller@neb.rr.com

H.B. Rosenstock

Solid State Division, Naval Research Laboratory

Follow this and additional works at: https://digitalcommons.unl.edu/physicsfuller

Part of the Physics Commons

Fuller, Robert and Rosenstock, H.B., "Equilibrium concentration of impurity-vacancy complexes" (1969). Robert G. Fuller Publications and Presentations. 26.

https://digitalcommons.unl.edu/physicsfuller/26

This Article is brought to you for free and open access by the Research Papers in Physics and Astronomy at DigitalCommons@University of Nebraska - Lincoln. It has been accepted for inclusion in Robert G. Fuller Publications and Presentations by an authorized administrator of DigitalCommons@University of Nebraska - Lincoln. 


\title{
Equilibrium concentration of impurity-vacancy complexes
}

\author{
R. G. Fuller \& H. B. Rosenstock \\ Solid State Division, Naval Research Laboratory
}

In recent years the transport properties of alkali halide crystals have been discussed in terms of five defects, i.e. isolated anion vacancies, isolated cation vacancies, isolated impurity ions, nearest neighbor vacancies of opposite electric charge (divacancies), and nearest neighbor impurity-vacancy complexes [1]. Originally these defects were treated as noninteracting particles located at appropriate lattice sites (the presence of divacancies and complexes was not assumed) and the defect concentrations were obtained from statistical mechanics. Subsequently the defects were allowed to interact with oppositely charged defects located at nearest neighbor lattice sites and the concentrations of divacancies and complexes were included in the analysis of experimental data. In 1954, Lidiard improved the theory of ionic conductivity by including the long-range Coulomb interactions between isolated defects [2]. Lidiard obtained closed-form equations for ionic conductivity by using the Debye-Hückel approximation for the Coulomb interactions. Recently these equations have become more widely used in the analysis of ionic conductivity data [35]. Now nonrandom deviations between the Lidiard-Debye-Hückel equations (LDH) and experimental ionic transport data have been reported [4-6]. This has on the one hand led to speculation about other defects being important in ionic transport phenomena. Cation Frenkel defects [46] and cation trivacancies [5-7] have both been discussed in recent conductivity and diffusion papers. On the other hand, the deviations between experiments and the LDH equations may arise from the assumptions and approximations implicit in those equations [8,9]. In particular, the LDH derivation [2] assumes that the Helmholtz free energy of an alkali halide crystal can be written as the sum of two parts: (a) a configurational term directly dependent on the presence of impurities and (b) a vibrational term which is independent of the arrangement of the impurities and vacancies in the lattice. This paper presents an alternative to the assumption that the vibrational term of the free energy is independent of the arrangement of the impurities and vacancies.

We begin by dealing with the cation sublattice of an alkali halide crystal containing $n_{i}$ impurity ions and $n_{c}$ vacant lattice sites. The impurities and vacancies are assumed to interact with energy $-B$ and hence form a number $n_{k}$ of bound impurity-vacancy complexes. We further assume that there is a definite change in vibrational motion when an impurity moves from an isolated site to a bound site near a vacancy. We treat this change in vibrational motion in the Einstein approximation, i.e. each impurity ion is an independent oscillator with the same frequency, but a different frequency is assigned to the bound impurity ions (complexes) than to the isolated impurity ions. The isolated impurity ions vibrate in shallow potential wells with energy level spacings of $\hbar \omega_{L}$, and the tightly bound impurity ions vibrate in deep potential wells with energy level spacings of $\hbar \omega_{k}[10]$.

In general, when $c_{j}$ states of energy $\varepsilon_{j}$ are available for $\mathrm{N}$ particles subject to Boltzmann statistics the number found in the $j$ th state is given by [11]

$$
n_{j}=c_{j} \exp \left(-\alpha-\beta \epsilon_{j}\right) .
$$

In the present situation this becomes 


$$
\left(n_{k}\right)_{j}=\left(c_{k}\right)_{j} \exp \left(-\alpha-j \beta\left(-B+\hbar \omega_{k}\right)\right)
$$

and

$$
\left(n_{i}-n_{k}\right)_{j}=\left(c_{L}\right)_{j} \exp \left(-\alpha-j \beta \hbar \omega_{L}\right)
$$

with $c_{k}=Z n_{c}$ where $Z$ is the number of possible bound sites near a vacancy and $n_{c}$ is the number of cation vacancies and $c_{L}=N-Z n_{c}$ where $N$ is the total number of sites available. When equations (2) and (3) are summed over $j$ the following results are obtained.

$$
\begin{gathered}
n_{k}=Z n_{c} \mathrm{e}^{-\alpha} \mathrm{e}^{\beta B}\left(1-\mathrm{e}^{-\hbar \omega_{k} \beta}\right)^{-1} \\
n_{i}-n_{k}=\left(N-Z n_{c}\right) \mathrm{e}^{-\alpha}\left(1-\mathrm{e}^{-\hbar \omega_{L} \beta}\right)^{-1}
\end{gathered}
$$

where $\beta$ is identified as $(k T)^{-1}$. Then equations (4) and (5) can be cast into a form similar to the usual law of mass action equation for complex concentration,

$$
\begin{aligned}
\frac{\left(N-Z n_{c}\right) n_{k}}{\left(n_{i}-n_{k}\right) n_{c}} & =Z \exp (B / k T) \\
& \times\left[\frac{1-\exp \left(-\hbar \omega_{L} / k T\right)}{1-\exp \left(-\hbar \omega_{k} / k T\right)}\right] .
\end{aligned}
$$

The term in square brackets results from allowing the crystal energy to be dependent upon the arrangements of the impurities in the lattice. The LDH result is readily incorporated into our result by replacing the $\exp (B / k T)$ term in equation (6) by the more complete expression from the LDH equation (See for example equation (12) [2]).

An illustration of the usefulness of equation (6) can be found in the analysis of the temperature dependence of the ionic conductivity of an alkali halide crystal containing a divalent-cation impurity. For example, an analysis of the conductivity data for a $\mathrm{KCl}: \mathrm{SrCl}_{2}$ crystal containing $375 \mathrm{ppm}$ mole fraction of strontium has already been reported[5]. The data was fitted to the LDH equations by the method of least squares with eleven adjustable parameters. In that analysis values were obtained for a jump attempt frequency $\omega^{-}$, for an impurity-vacancy binding energy $B$ expressed as an enthalpy minus the temperature-entropy product. and for the cation migration enthalpy and entropy.

To compare least square analyses of experimental data using two different sets of theoretical equations. i.e. the $\mathrm{LDH}$ equations and the $\mathrm{LDH}$ equations modified by equation (6), it is desirable to use the same number of adjustable parameters in each analysis. So values for the quantities in equation (6) that do not appear in the LDH equations were estimated in terms of LDH parameters. These estimates were obtained from Einstein oscillator models of the isolated and bound impurity ions. The jump attempt $\bar{\omega}$ as determined from the LDH analysis of the conductivity was taken as the average of the potassium and chlorine ion vibration frequencies and was related to the masses of the potassium $\left(M_{\mathrm{K}}\right)$ and chlorine $\left(M_{\mathrm{Cl}}\right)$ ions by the following equations:

$$
\bar{\omega}=\frac{1}{2}\left(\omega_{\mathrm{K}}+\omega_{\mathrm{Cl}}\right) \propto \frac{1}{2}\left(M_{\mathrm{K}}^{-1 / 2}+M_{\mathrm{Cl}}^{-1 / 2}\right),
$$

and the Einstein frequency $\omega_{L}$ for an isolated $\mathrm{Sr}$ ion was estimated to be

$$
\omega_{L}=\frac{2 M_{\mathrm{K}}^{1 / 2} M_{\mathrm{Cl}}^{1 / 2} \bar{\omega}}{M_{\mathrm{Sr}}^{1 / 2}\left(M_{\mathrm{K}}^{1 / 2}+M_{\mathrm{Cl}}^{1 / 2}\right)}=0.65 \bar{\omega}
$$

The binding energy $B$ as determined from the least squares analysis was related to $\omega_{k}$ and $\omega_{L}$ in a simple harmonic approximation. It seems reasonable that the particles in deeper traps would vibrate with higher frequency. To simply estimate the frequency difference let us assume that both bound and isolated impurity ions oscillated with a maximum displacement of $a / 2$. The two oscillators were taken to have force constants of $M_{\mathrm{Sr}} \omega_{k}^{2 / 2}$ and $M_{\mathrm{Sr}} \omega_{L}^{2 / 2}$ respectively. Furthermore, the lowest energy levels of the two oscil- 


\section{Table 1. Results of analyses of conductivity of $\mathrm{KCl}: \mathrm{SrCl}_{2}$ crystal containing $375 \mathrm{ppm} \mathrm{Sr}$}

\begin{tabular}{lcc}
\hline \multicolumn{1}{c}{ Quantity } & $\begin{array}{c}\text { Our result LDH theory } \\
\text { modified by equation (6) }\end{array}$ & LDH theory [5] \\
\hline Cation vacancy migration enthalpy (eV) & $0 \cdot 74$ & $0 \cdot 74$ \\
Cation vacancy migration entropy & $2 \cdot 4 k$ & $2 \cdot 4 k$ \\
Divalent-cation-impurity-cation- & $0 \cdot 66$ & $0 \cdot 60$ \\
vacancy binding enthalpy (eV) & & $2 \cdot 3 k$ \\
Divalent-cation-impurity-cation- & $2 \cdot 3 k$ & $37 \cdot 7 \times 10^{12}$ \\
vacancy binding entropy & $37 \cdot 1 \times 10^{12}$ & $0 \cdot 67 \%$ \\
Jump attempt frequency $\bar{\omega}\left(\mathrm{sec}^{-1}\right)$ & $0 \cdot 33 \%$ & \\
Average rms fractional deviation & & \\
\hline
\end{tabular}

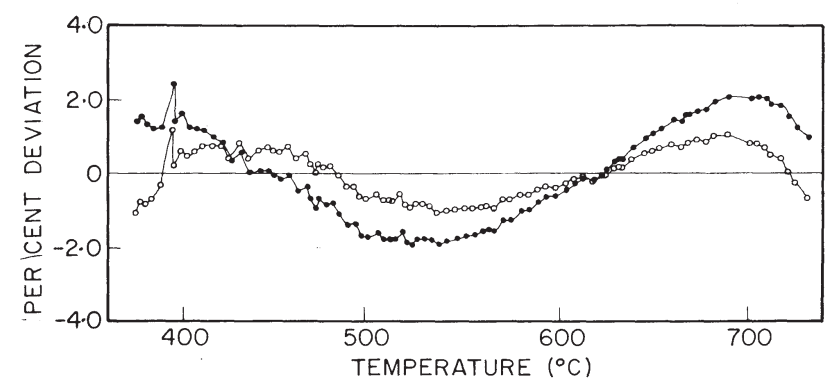

Fig. 1. Per cent deviation as a function of temperature for the $\mathrm{KCl}$ crystal containing $375 \mathrm{ppm}$ Sr. 0 . per cent deviation between theoretical and experimental values of conductivity with Lidiard-Debye-Hückel theory; O, per cent deviation between theoretical and experimental values of conductivity with the Lidiard-Debye-Huickel theory modified by the square bracketed term in equation (6).

lators differ by an energy of $B[10]$. On the basis of such a model the following relationship can be derived for $\omega_{k}$ and $\omega_{L}$ :

$$
\omega_{k}^{2}=\omega_{L}^{2}+\gamma B
$$

where

$$
\gamma=8 / M_{\mathrm{Sr}} a^{2}
$$

A value of $207 \times 10^{26} \mathrm{sec}^{-2} \mathrm{eV}^{-1}$ for $\gamma$ gave the best fit to the experimental data. This leads to $a / 2$ $=0 \cdot 10 \AA$, a reasonable value for the maximum displacement amplitude, and to $\omega_{k}=26.6 \times 10^{12}$ $\mathrm{sec}^{-1}$ at room temperature as compared to $24.1 \times$ $10^{12} \mathrm{sec}^{-1}$ for $\omega_{L}$.
The results of analyses before and after inclusion of equation (6) are shown in terms of parameter values in Table 1 and in terms of the per cent deviation between theoretical and experimental values of conductivity as a function of temperature in Fig. 1. (The complete discussion of the computer analysis procedure is contained in [5].) The theoretical values of ionic conductivity calculated with the LDH equations modified by equation (6) do provide the better fit to the experimental conductivity data, and reduce the rms percent deviation from 0.67 to 0.33 per cent.

In summary an equation for the equilibrium concentration of impurity-vacancy complex- 
es has been derived. This derivation has included the dependence of the vibrational term of the crystal free energy upon the arrangement of the impurity ions. The vibrational changes in a simple Einstein approximation have been incorporated into the equation for complex concentration. It is encouraging to note that even in this simple approximation the resulting equations for ionic transport are more in accord with experimental data than transport equations which neglect the vibrational effects of the arrangement or the impurity ions.

Acknowledgements - The authors gratefully acknowledge helpful discussions with Drs. Manuel Gomez and Michael H. Reilly.

\section{R. G. FULLER}

\section{H. B. ROSENSTOCK}

Solid State Division, Naval Research Laboratory, Washington, D.C. 20390 U.S.A.

\section{REFERENCES}

1. SUPTITZ P. and TElTOW, J., Phys. Status Solidi 23, 9 (1967).

2. LIDIARD A. B., Phys. Rev. 94, 29 (1954).

3. KANZAKI H., KIDO K. and TAMURA S., J. Phys. Soc. Japan 20, 2305 (1965).

4. ALLNATT A. R. and PANTELIS P., Solid State Comm. 6, 309 (1968).

5. FULlER R. G., MARQUARDT C. L., REILlY M. H. and WELlS J. C., Phys. Rev. 176, 1036 (1968).

6. NELSON V. C. and FRIAUF R. J., U.S. Atomic Energy Commission, Tech. Rep. COO-1197-32, The University of Kansas (1968).

7. FUller R. G. and ReIlly M. H., J. Phys. Chem. Solids 30, 457 (1969).

8. AllnatT A. R. and COHEN M. H., J. Chem. Phys. 40,1860 (1964).

9. AllnatT A. R. and COHEN M. H., J. Chem. Phys. 40, 1871 (1964).

10. Here $B$ represents the energy between the lowest vibrational levels and is less than the binding energy defined in other papers (See FULLER R. G., MARQUARDT C. L., REILlY M. H. and WELlS J. C., Phys. Rev. 176, 1036 (1968).) by $\hbar / 2\left(\omega_{k}-\omega_{L}\right)$, the zero point energy terms.

11. MAYER J. E. and MAYER M. G., In Statistical Mechanics, p. 114. Wiley, New York (1940). 